Curr Opin Ophthalmol. 2017 November ; 28(6): 629-635. doi:10.1097/ICU.0000000000000421.

\title{
Tubulointerstitial Nephritis and Uveitis
}

\author{
Kaivon Pakzad-Vaezi ${ }^{1}$ and Kathryn L Pepple ${ }^{1}$ \\ ${ }^{1}$ Department of Ophthalmology, University of Washington
}

\begin{abstract}
Purpose of review-Tubulointerstitial nephritis and uveitis (TINU) is an important yet underrecognized ocular inflammatory syndrome. This review summarizes key historical publications that identified and defined the syndrome, and more recent literature that reveal the importance of urinary beta-2 microglobulin testing and kidney biopsy in the diagnostic evaluation of patients with TINU. Additionally, research studies providing new insights into disease pathogenesis are highlighted.
\end{abstract}

Recent findings-In contrast with initial reports of TINU manifesting exclusively as an anterior uveitis in pediatric patients, more recent reports have identified TINU in patients of all ages with a wide range of ocular manifestations. Urinary beta- 2 microglobulin has emerged as a sensitive and specific laboratory screening test, and the role of kidney biopsy in differentiating TINU from sarcoidosis continues to evolve. Genetic studies have identified HLA-DQA $1 * 01$, HLA-DQB $1 * 05$, and HLA-DRB $1 * 01$ as high risk alleles and the identification of anti-mCRP antibodies suggests a role for humoral immunity in disease pathogenesis. Management strategies have evolved to include systemic anti-inflammatory treatment as a result of important outcome studies in patients with significant renal and ocular disease.

Summary-With greater recognition, understanding, and treatment of this syndrome, both ocular inflammation and renal disease can be better addressed.

\section{Keywords}

Uveitis; tubulointerstitial nephritis; tubulointerstitial nephritis and uveitis; sarcoidosis; review

\section{Introduction}

Since the first description in 1975, tubulointerstitial nephritis and uveitis syndrome (TINU) has become increasingly recognized as not only an important cause of uveitis in the pediatric and young adult age-groups, but also in older adults [1-3*]. It comprises approximately 1$2 \%$ of cases in subspecialty practices, but may represent up to a third of bilateral acute-onset anterior uveitis in patients younger than 20 [2,4-7**]. Correctly identifying patients with TINU provides an opportunity for ophthalmologists to collaborate with colleagues in pediatrics, internal medicine, and nephrology to prevent permanent ocular and renal damage.

Corresponding Author: Kathryn L Pepple, 908 Jefferson St, $7^{\text {th }}$ floor, Harborview Medical Center, University of Washington, Seattle, WA 98104, kpepple@uw.edu, Phone: 206-616-1292, Fax: 206-685-7055.

Conflicts of interest: KPV - none. KLP - none. 


\section{Presentation}

Classically, TINU presents as a symptomatic (redness, pain, photophobia), bilateral, acuteonset non-granulomatous anterior uveitis in children that is associated with the diagnosis of interstitial nephritis $[2,5,8]$. Tubulointerstitial nephritis typically precedes or coincides with ocular disease, and is usually more severe in older age-groups [2,5]. Interstitial nephritis is usually associated with systemic symptoms such as fever, weight loss, and fatigue, but may be asymptomatic. The renal course is thought to be independent from ocular disease as neither the severity nor the prognosis of nephritis is influenced by the presence of uveitis $\left[3^{*}, 9\right]$. Asymptomatic uveitis in the context of pediatric tubulointerstitial nephritis may be under-recognized, with one prospective study identifying uveitis in $50 \%$ of patients with biopsy-proven interstitial nephritis in [10]. However, no guidelines or recommendations for proactive ocular screening in patients with interstitial nephritis have been made to date. TINU has not been identified with higher frequency in any ethnic group [5], and gender is no longer believed to contribute to disease predisposition $\left[2,3^{*}, 5,6^{* *}, 8\right]$. In addition to bilateral anterior chamber cell and flare, posterior synechiae, cystoid macular edema, disc edema (especially in the young), elevated intraocular pressure, and cataract are common ocular complications found in patients with TINU and may be seen at presentation [2,5,11].

Subsequent to initial descriptions, reports of TINU cases of many ages [2,3*] with a broad range of ocular manifestations including intermediate and panuveitis has challenged early classification $\left[2,3^{*}, 5,6^{* *}, 12-14^{* *}\right]$. Some of the first reports of posterior segment involvement included isolated macular and disc edema [5], which are manifestations that would not be classified as posterior uveitis under the Standardization of Uveitis Nomenclature (SUN) [15]. However, more recent reports using SUN criteria have identified TINU patients with intermediate and panuveitis $\left[3^{*}, 6^{* *}, 14^{* *}\right]$ suggesting that these presentations could have been previously underappreciated. Other rare descriptions of posterior findings in the most recent literature include small hypopigmented chorioretinal scars [14**,16], delayed multifocal choroiditis [17], acute posterior multifocal placoid pigment epitheliopathy [18], choroidal neovascular membranes [19], and neuroretinitis $[14,20]$. Atypical anterior findings such as granulomatous keratic precipitates and iris nodules have also been described in cases of TINU [5]. While the classic presentation of TINU as a nongranulomatous anterior uveitis will likely remain the predominant manifestation, it is clear that in this syndrome a spectrum of signs is possible. It should be remembered, however, that TINU is a diagnosis of exclusion, and the investigation of other uveitic causes that more typically cause the above findings, particularly sarcoidosis, is required.

\section{Diagnosis}

TINU is rare, being diagnosed in less than $2 \%$ (range 1-2\%) of all patients seen in tertiary care uveitis settings $[2,21,22]$. However, when considering the subset of patients with bilateral acute anterior uveitis, TINU will be identified in up to $10 \%$ of patients. This percentage increases in the pediatric population, with TINU identified in approximately $1 / 3$ of patients less than 20 years of age with bilateral sudden-onset anterior uveitis [2]. 
Diagnostic criteria for TINU were published in 2001 based on review of 133 cases published until that point [5]. These criteria provided levels of certainty ranging from "definite", "probable", and "possible" (Table). Due to the specific definition of "typical uveitis" required for definite TINU, the long-term utility of this system is limited. However, the criteria used for diagnosing nephritis are instructive. While renal biopsy is considered the gold standard for diagnosing interstitial nephritis, "definite" TINU can be diagnosed in its absence as long as key clinical findings are present. Despite the vague nature of the term "systemic illness" (Table), when such symptoms are present in the context of abnormal renal function and urinalysis results consistent with the diagnosis, many nephrologists will consider this sufficient evidence to confirm the diagnosis [5]. Laboratory testing abnormalities in TINU patients can include elevated serum creatinine (55-90\% of patients), and low-grade proteinuria, microscopic hematuria, and leukocytes by urinalysis [2,5]. Urinary beta- 2 microglobulin $(\beta 2 \mathrm{M})$ levels are elevated in over $87 \%$ of cases [4-7**], and can be elevated in the absence of abnormal renal function [8]. Elevated serum erythrocyte sedimentation rate, immunoglobulin G, and decreased hematocrit may also be found [5], though these tests can be abnormal in a wide variety of diseases and are not sensitive or specific for TINU.

Elevated urinary $\beta 2 \mathrm{M}$ has emerged as a sensitive and specific non-invasive test useful in the diagnosis of TINU [7,14**]. $\beta 2 \mathrm{M}$ is a small $(12-\mathrm{kDa})$ protein excreted by glomeruli and resorbed by healthy tubular epithelium. This resorption is reduced in tubulointerstitial nephritis, leading to elevated levels in the urine [6**]. Levels can correlate to histologic grade of interstitial nephritis [7], and can remain elevated after renal function normalizes, making it a useful test for previous renal dysfunction [5,7,8]. However, it can be elevated in any cause of interstitial nephritis and is not specific for TINU. Hettinga et al [6**] studied the utility of $\beta 2 \mathrm{M}$ measurement in 45 consecutive new patients under the age of 23 sent to their referral center with uveitis of any type. Overall, urine $\beta 2 \mathrm{M}$ was elevated in $40 \%$ of patients, serum creatinine was elevated in $22 \%$, and $47 \%$ had pyuria. In total, two patients were diagnosed as definite TINU based on renal biopsy, while 6 patients were diagnosed as probable TINU based on clinical and laboratory criteria. Urine $\beta 2 \mathrm{M}$ was elevated in 7 of these 8 patients. The median $\beta 2 \mathrm{M}$ level was $1,950 \mu \mathrm{g} / \mathrm{L}$ (95\% confidence interval (CI): $1,260-5,160 \mu \mathrm{g} / \mathrm{L}$ ) in the TINU cases, compared to $200 \mu \mathrm{g} / \mathrm{L}$ (95\% CI: $190-210 \mu \mathrm{g} / \mathrm{L}$ ) in cases with normal renal function. Of the 11 other patients with elevated $\beta 2 \mathrm{M}$ who were not diagnosed with TINU (most having idiopathic uveitis), only one patient had a $\beta 2 \mathrm{M}$ value greater than $1,000 \mu \mathrm{g} / \mathrm{L}$, while the rest were between $200-1,000 \mu \mathrm{g} / \mathrm{L}$. Stated another way, while 39\% (7/18) of patients with abnormal $\beta 2 \mathrm{M}$ in total were diagnosed as TINU, $86 \%$ (7/8) of those with $\beta 2 \mathrm{M}$ greater than $1,000 \mu \mathrm{g} / \mathrm{L}$ had TINU, while none $(0 / 10)$ with $\beta 2 \mathrm{M}$ between $200-1,000 \mu \mathrm{g} / \mathrm{L}$ did. The sensitivity and specificity of elevated $\beta 2 \mathrm{M}$ was $87.5 \%$ and $70 \%$, respectively, with positive (PPV) and negative predictive values (NPV) of urinary $\beta 2 \mathrm{M}$ greater than $1,000 \mu \mathrm{g} / \mathrm{L}$ being $88 \%$ and $97 \%$, respectively. While the combination of elevated serum creatinine and urine B2M yielded a PPV of 100\%, concern arises regarding the contribution of serum creatinine in these calculations, as it was used as a diagnostic criteria required in the definition of TINU. This study only evaluated urinary $\beta 2 \mathrm{M}$ in patients younger than 22 , so it remains to be seen if these results are generalizable to older patients. 
In summary, as part of the TINU laboratory evaluation, in addition to studies to rule out differential diagnoses, there is sufficient evidence to support ordering a urinalysis, a urinary $\beta 2 \mathrm{M}$, and serum creatinine. The presence of a normal urinary $\beta 2 \mathrm{M}$ is helpful to rule TINU out, while the creatinine and urinalysis will be important information to guide nephrology management in the case of an elevated urinary $\beta 2 \mathrm{M}$. Further workup of an elevated urinary $\beta 2 \mathrm{M}$ includes referral to a nephrologist for consideration of renal biopsy and to determine renal indications for systemic treatment.

\section{Differential diagnosis}

Other important diagnoses to consider include those that may manifest both uveitis and interstitial nephritis. In particular, these include sarcoidosis, systemic lupus erythematosis, Sjogren's syndrome, granulomatous polyangiitis, Behcet's disease, tuberculosis, and syphilis [5,23]. Most of these will not cause the anterior uveitis typical of TINU, and often demonstrate different characteristic systemic symptoms and signs that are readily distinguishable from TINU. Serologic testing for these conditions should be guided based on a complete review of systems and clinical examination.

Sarcoidosis can be particularly challenging to rule out, particularly in the pediatric population where it can present without the typical pulmonary manifestations. In cases where renal biopsy is performed, the pathology findings will typically clarify the diagnosis. Granulomas are rare in patients with isolated interstitial nephritis [24]. Renal biopsies obtained in cases of interstitial nephritis that are not associated with uveitis contain granulomas less than $1 \%$ of the time [24]. Granulomas are also an uncommon finding in biopsies from patients with TINU. In the Mandeville review [5] including 118 biopsied TINU cases before 2001, only 15 patients (13\%) had evidence of non-caseating granulomas on renal biopsy. Interestingly, almost half of these patients also had granulomas found on bone marrow or liver biopsies [5]. This evidence of more widespread granulomas suggests sarcoidosis could have been considered as a confounding differential in these cases as well. While granulomas appear to be the exception in cases of TINU, they are the defining lesion in sarcoidosis. Sarcoidosis commonly affects the eye, and approximately $20 \%$ of patients with systemic sarcoidosis will develop granulomatous interstitial nephritis [25,26]. Thus when biopsies identify renal granulomas, and particularly if there is evidence of other systemic granulomatous disease, sarcoidosis rather than TINU is the preferred diagnosis. Furthermore, in patients with TINU where granulomas are identified on biopsy, there should be increased vigilance to monitor for possible granulomatous disease in other organ systems.

Since granulomas can be present in cases of true TINU, a close review of the pathology specimen in conjunction with pathology and nephrology may be required to determine the most appropriate diagnosis. This combined approach was utilized in the case of a 9 year-old male with bilateral chronic anterior uveitis and macular edema poorly responsive to topical steroids that was sent for renal biopsy as part of the evaluation for TINU. His urinary $\beta 2 \mathrm{M}$ was extremely elevated, 85-fold greater than the upper limit of normal, and 5-fold greater the highest values usually seen in TINU [6**,27*]. Renal biopsy revealed interstitial nephritis and a significant burden of non-caseating granulomas that was more in line with the diagnosis of renal and ocular sarcoidosis rather than TINU [27*]. However, there are grey 
areas that even a biopsy can have difficulty clarifying. For example, another recent case report of a 22 year-old male with a bilateral, granulomatous, and hypertensive anterior uveitis, resulted in the diagnosis of TINU despite the presence of numerous non-caseating granulomas associated with interstitial nephritis on renal biopsy [28]. These cases highlight two important points: TINU is a diagnosis of exclusion, and that renal biopsy can help provide diagnostic clarity in challenging cases $[26,29,30]$.

\section{Pathogenesis}

The pathogenesis of TINU is likely multifactorial, with contributions from genetic, infectious, autoimmune, and iatrogenic factors $\left[5,6^{* *}, 31^{* *}, 32\right]$. The nephrology literature has made the most headway in exploring the links between these factors and acute interstitial nephritis (reviewed in [31**]). Work in this field has identified a role for allergy-mediated immune mechanisms in drug-induced interstitial nephritis, and mutations in genes encoding the "tubulointerstitial nephritis antigen" leading to chronic disease [31**]. However, the mechanistic links underlying the ocular disease, or tying together the nephritis and uveitis remain largely unknown. Two promising areas of investigation are focused on understanding the dysregulation of cell-mediated immunity $[7,33,34]$ and humoral immunity in patients with TINU [35-37].

Loss of T-cell tolerance in the pathogenesis of TINU is suggested by multiple studies identifying a strong link between TINU and certain class II human leukocyte antigen (HLA) subtypes, such as HLA-DQA $1 * 01$, HLA-DQB $1 * 05$, and HLA-DRB $1 * 01$, with a relative risk as high as 167.1 for HLA-DRB $1 * 0102[12,13,38]$. The latter HLA gene is an independent risk factor for TINU, particularly of younger onset [38]. These HLA subtypes confer variable risks based on the population studied $[13,39,40]$. Since HLA class II molecules are involved with exogenous antigen presentation to CD4+ T-helper cells [41], molecular mimicry between exogenous infectious antigens and ocular antigens could explain the HLA association with this disease [42]. Other potential mechanisms could include an insufficient or poorly functioning antigen-specific regulatory T-cell population $[41,43]$. However, there is controversy about the role of regulatory T-cells in autoimmune uveitis $[44,45]$, and no TINU-specific studies have been reported.

The role of humoral immunity in TINU pathogenesis has been suggested by the identification of autoreactive antibodies directed against renal and ocular antigens in TINU patients [35,36]. One study identified elevated titers of anti-monomeric C-reactive protein (anti-mCRP) antibodies in the serum of 9 patients with active TINU [36]. Serum from healthy subjects, and from active patients with isolated acute interstitial nephritis (AIN, idiopathic or drug-related), ANCA-vasculitis, IgA-nephropathy, minimal change disease, Sjogren's syndrome, or amyloidosis was also tested. This study found that elevated titers of anti-mCRP antibodies were not unique to patients with TINU, but the percentage of patients with elevated titers was significantly higher in the TINU group $(9 / 9,100 \%)$ when compared to all other groups. The only disease category with more than 2 patients that also demonstrated an elevated titer was AIN (4/11,36\%). It is not known if these patients with AIN were screened for eye disease, their follow-up course, or if any did ultimately proceed to TINU, but it is interesting that other forms of kidney disease other than interstitial 
nephritis did not lead to the development of anti-mCRP antibodies. This suggests that these antibodies may have disease-specific relevance. In addition to elevated serum anti-mCRP antibodies, the authors also showed an increase in the extent of mCRP found in kidney biopsies by immunohistochemistry when compared to normal kidney. In the eye, mCRP was identified in normal human iris and ciliary body collected at the time of trabeculectomy by immunohistochemistry. Serum obtained from patients with active TINU colocalized with mCRP staining in these normal tissues further suggesting a mechanistic link between this protein and disease pathogenesis. $\mathrm{mCRP}$ is the dissociated monomer of the parent acute phase reactant pentamer (C-reactive protein), and is involved in the activation and regulation of the complement pathway, but no tissue specific role for the protein has been identified [46]. Therefore, it is not clear if mCRP is truly a specific antigen involved in ocular and renal disease, or if these findings represent an auto-antibody that develops to this acute phase reactant as a results of chronic inflammation as has been reported previously in lupus nephritis [47]. Understanding the role of humoral immunity in uveitis has become more important as we try to explain the success of rituximab, a B-cell depleting biologic therapy, on certain forms of recalcitrant uveitis $[48,49]$. A more thorough understanding of TINU pathogenesis may also uncover an important role for humoral immunity in other forms of uveitis.

Another interesting question in TINU pathogenesis is whether the disease is a sequential process where an initial renal (or potentially uveal) insult incites an inflammatory cascade with secondary effects on the other organ, or if both tissues share a common target for a single underlying mechanism. However, there is evidence to suggest that the kidney is the primary target. One study found that in patients with AIN, elevated anti-mCRP antibodies were predictive of subsequent uveitis development [37]. Thus, a reasonable hypothesis for disease pathogenesis begins with exposure of the kidney to an inciting agent that stimulates a HLA class-II response that targets the immune system to a common antigen in both organs. Most likely this antigen is a native protein in the uvea and renal interstitium that shares a common epitope. However, the mCRP data also suggests the antigen could be an acute phase reactant that deposits in the eye and kidney. Experimental animal models will be required to address these possible hypotheses.

\section{Prognosis and Treatment}

With treatment, ocular and renal outcomes for patients with TINU are usually good. Particularly for "classic" TINU patients, most will maintain or improve vision from presentation and as a cohort achieve an average vision of 20/25 or better [2]. Ocular inflammation and renal function respond promptly to corticosteroid treatment during active disease, but maintaining quiescence in the eye can be more difficult than in the kidney. Ocular disease recurs in up to $50 \%$ of patients after corticosteroid withdrawal, and chronic uveitis develops more frequently in pediatric patients than in adults [5]. Thus, although longterm outcomes are generally good for both the eye and kidney, ocular disease frequently determines the need for ongoing systemic therapy. It is not clear if this is a manifestation of more severe or aggressive disease in the eye or more sensitive measures of end-organ impact available to the ophthalmologist (e.g. ability to count individual cells at the slit-lamp). 
In the acute phase, around $1 / 3$ of patients with TINU who have anterior uveitis will respond to topical corticosteroids, while $2 / 3$ will require systemic corticosteroids (median duration of treatment 2.5 months) [2,5,31**]. In bilateral, intermediate, or posterior disease, systemic corticosteroids are typically required with the option for local steroid injections reserved for the rare unilateral case. Earlier studies reported high rates of spontaneous resolution, with only $10 \%$ of patients requiring chronic steroid-sparing immunomodulation therapy (IMT) [5]. However, in light of more recent reports, those studies likely underestimated the need for chronic therapy due to their short follow-up and/or management by non-uveitis specialists [14**]. Younger age has been identified as a risk factor for developing chronic disease, but few studies have specifically looked at the long-term risk of recurrence in patients with TINU, and the impact of systemic therapy on reducing that risk. The concern that this population is at risk for undertreatment was further raised by a recent series of 9 patients that were followed for more than 3 years (median follow-up time) after their initial diagnosis and treatment. The authors found inflammation recurred after corticosteroid taper in 56\% of patients, prompting the use of IMT. However, with at least 12 months of adequate control (mean: 29.5 months; range: 13-40 months), uveitis recurrences were substantially reduced upon treatment withdrawal [14**]. Because their goal was aggressive and durable inflammation control, these authors routinely started IMT early in the treatment course. Thus whether the durable remission was due to early IMT or aggressive inflammatory control regardless of the treatment regimen was not addressed and requires further study.

In the absence of an ocular indication for systemic therapy, some patients will still require systemic corticosteroids or immune modulation for their renal disease. Early TINU literature held that renal disease often resolved spontaneously, and recommended reserving systemic therapy for evidence of progressive renal disease $[5,7,50]$. This practice has become less common due to reports of continued nephritis on repeat biopsy after pulse corticosteroids without long-term treatment [51,52]. In addition to longer therapy, timing of therapy is also a consideration. In one series, prompt corticosteroid therapy greatly improved short-term urinary $\beta 2 \mathrm{M}$ levels and inflammatory signs on repeat biopsy in 3 patients, while a fourth patient with delayed treatment demonstrated persistent elevations in $\beta 2 \mathrm{M}$ and renal inflammation, with subsequent permanent renal damage [53]. Despite these data suggesting benefit to more frequent use of corticosteroids, one prospective and one retrospective study from the nephrology literature failed to identify differences in final renal function at 6 months and 1 year, respectively, in patients who were treated compared to those who are not, despite more rapid recovery in those who were treated $[3 *, 9]$. These data are difficult to interpret as they arise from small studies, have difficulties controlling for the time of true renal disease onset, and may reflect a more kidney-dominant version of the disease than typically seen initially by the ophthalmologist. Although unusual, severe TINU can lead to significant renal compromise requiring dialysis and kidney transplant $\left[2,3^{*}, 5,54\right]$. This further supports the importance of collaborating with nephrology colleagues for case-bycase management decisions and to ensure patients are benefitting from the most current treatment guidelines. Fortunately, however, once in remission (spontaneous or after treatment), renal recurrences rarely occur $\left[2-5^{*}, 10,31^{* *}\right]$.

Specific conclusions about how management of TINU should differ from the standard approach to uveitis therapy are challenging based on the quality of current evidence. Case 
series are small in size given the rarity of this condition, and present variable primary endpoints based on treating subspecialty (ophthalmology versus nephrology) and patient age-group reported (pediatric versus adult). To date, no large randomized controlled trials have been conducted to identify optimal treatment paradigms, and no cohort studies are available to clarify the natural history of TINU. We recommend an approach to uveitis management in TINU following commonly accepted expert guidelines [55] that utilize escalating topical and/or systemic therapy depending on the location of uveitis and its response to treatment. We institute IMT as required for improved anti-inflammatory control, to reduce recurrences upon steroid tapering, or to reduce unwanted steroid-related adverse effects, and seek to maintain quiescence for at least 12-24 months before withdrawing treatment. In addition, we routinely involve nephrology early in order to assist with both diagnosis confirmation and management of renal disease.

\section{Conclusion}

Although rare, the diagnosis of TINU may have important systemic implications for both pediatric and adult patients. It is important for the ophthalmologist to consider testing urinary $\beta 2 \mathrm{M}$ in cases of simultaneous or sequential symptomatic bilateral anterior uveitis. Nephrology consultation and possible renal biopsy are important follow-up steps when abnormalities are found, even if ocular disease is responding to local therapy. While variable clinical presentations are possible in patients with TINU, the importance of excluding other diagnoses in the differential, particularly sarcoidosis, must be considered. The ocular and renal prognosis is usually good, and disease control is usually prompt in response to topical and systemic steroids. However, uveitis recurrences are common after corticosteroid withdrawal and chronic kidney damage can be an underappreciated problem in the absence of monitoring and collaboration with other specialists.

\section{Acknowledgments}

None

Financial support and sponsorship:

This study was supported by an unrestricted grant from Research to Prevent Blindness (New York, NY): NEI K08EY023998 (KLP).

\section{References}

1. Dobrin RS, Vernier RL, Fish AL. Acute eosinophilic interstitial nephritis and renal failure with bone marrow-lymph node granulomas and anterior uveitis. A new syndrome. Am J Med. 1975; 59:325333. [PubMed: 1163543]

2. Mackensen F, Smith JR, Rosenbaum JT. Enhanced recognition, treatment, and prognosis of tubulointerstitial nephritis and uveitis syndrome. Ophthalmology. 2007; 114:995-999. [PubMed: 17383731]

*3. Legendre M, Devilliers H, Perard L, et al. Clinicopathologic characteristics, treatment, and outcomes of tubulointerstitial nephritis and uveitis syndrome in adults: A national retrospective strobe-compliant study. Medicine (Baltimore). 2016; 95:e3964. *Recent study describing broad presentation of TINU in adults and both uveitis and renal outcomes of treatment. [PubMed: 27367994] 
4. Mackensen F, Billing H. Tubulointerstitial nephritis and uveitis syndrome. Curr Opin Ophthalmol. 2009; 20:525-531. [PubMed: 19752730]

5. Mandeville JT, Levinson RD, Holland GN. The tubulointerstitial nephritis and uveitis syndrome. Surv Ophthalmol. 2001; 46:195-208. [PubMed: 11738428]

**6. Hettinga YM, Scheerlinck LME, Lilien MR, et al. The value of measuring urinary $\beta 2$ microglobulin and serum creatinine for detecting tubulointerstitial nephritis and uveitis syndrome in young patients with uveitis. JAMA Ophthalmol. 2015; 133:140-145. **Analysis demonstrating utility of urinary beta- 2 microglobulin testing in TINU, particularly for ruling out the disease. [PubMed: 25356569]

7. Takemura T, Okada M, Hino S, et al. Course and outcome of tubulointerstitial nephritis and uveitis syndrome. Am J Kidney Dis. 1999; 34:1016-1021. [PubMed: 10585310]

8. Goda C, Kotake S, Ichiishi A, et al. Clinical features in tubulointerstitial nephritis and uveitis (TINU) syndrome. Am J Ophthalmol. 2005; 140:637-641. [PubMed: 16226516]

9. Jahnukainen T, Saarela V, Arikoski P, et al. Prednisone in the treatment of tubulointerstitial nephritis in children. Pediatr Nephrol. 2013; 28:1253-1260. [PubMed: 23605375]

10. Saarela V, Nuutinen M, Ala-Houhala M, et al. Tubulointerstitial nephritis and uveitis syndrome in children: a prospective multicenter study. Ophthalmology. 2013; 120:1476-1481. [PubMed: 23511116]

11. Sherman MD, Own KH. Interstitial nephritis and uveitis syndrome presenting with bilateral optic disk edema. Am J Ophthalmol. 1999; 127:609-610. [PubMed: 10334360]

12. Levinson RD, Park MS, Rikkers SM, et al. Strong associations between specific HLA-DQ and HLA-DR alleles and the tubulointerstitial nephritis and uveitis syndrome. Invest Ophthalmol Vis Sci. 2003; 44:653-657. [PubMed: 12556395]

13. Reddy AK, Hwang Y-S, Mandelcorn ED, Davis JL. HLA-DR, DQ class II DNA typing in pediatric panuveitis and tubulointerstitial nephritis and uveitis. Am J Ophthalmol. 2014; 157:678-686. [PubMed: 24321473]

**14. Sobolewska B, Bayyoud T, Deuter C, et al. Long-term follow-up of patients with Tubulointerstitial Nephritis and Uveitis (TINU) Syndrome. Ocul Immunol Inflamm. 2016; 12:17. **Patients with TINU may require more steroid-sparing immunosuppressive therapy than previous thought.

15. Jabs DA, Nussenblatt RB, Rosenbaum JT. Standardization of Uveitis Nomenclature (SUN) Working Group. Standardization of uveitis nomenclature for reporting clinical data. Results of the First International Workshop. Am J Ophthalmol. 2005; 140:509-516. [PubMed: 16196117]

16. Ali A, Rosenbaum JT. TINU (tubulointerstitial nephritis uveitis) can be associated with chorioretinal scars. Ocul Immunol Inflamm. 2014; 22:213-217. [PubMed: 24143861]

17. Derzko-Dzulynsky L, Rabinovitch T. Tubulointerstitial nephritis and uveitis with bilateral multifocal choroiditis. Am J Ophthalmol. 2000; 129:807-809. [PubMed: 10926997]

18. Lee AR, Sharma S, Mahmoud TH. Tubulointerstitial nephritis and uveitis syndrome with a primary presentation of acute posterior multifocal placoid pigment epitheliopathy. Retin Cases Brief Rep. 2017; 11:100-103. [PubMed: 26967965]

19. Takemoto Y, Namba K, Mizuuchi K, et al. Two cases of subfoveal choroidal neovascularization with tubulointerstitial nephritis and uveitis syndrome. Eur J Ophthalmol. 2013; 23:255-257. [PubMed: 23335312]

20. Khochtali S, Harzallah O, Hadhri R, et al. Neuroretinitis: a rare feature of tubulointerstitial nephritis and uveitis syndrome. Int Ophthalmol. 2014; 34:629-633. [PubMed: 23824660]

21. Rosenbaum JT. Bilateral anterior uveitis and interstitial nephritis. Am J Ophthalmol. 1988; 105:534-537. [PubMed: 3369519]

22. Kump LI, Cervantes-Castañeda RA, Androudi SN, Foster CS. Analysis of pediatric uveitis cases at a tertiary referral center. Ophthalmology. 2005; 112:1287-1292. [PubMed: 15921752]

23. Sessa A, Meroni M, Battini G, et al. Acute renal failure due to idiopathic tubulo-intestinal nephritis and uveitis: "TINU syndrome" Case report and review of the literature. J Nephrol . 2000; 13:377380. [PubMed: 11063143]

24. Perazella MA, Markowitz GS. Drug-induced acute interstitial nephritis. Nat Rev Nephrol. 2010; 6:461-470. [PubMed: 20517290] 
25. Göbel U, Kettritz R, Schneider W, Luft F. The protean face of renal sarcoidosis. J Am Soc Nephrol. 2001; 12:616-623. [PubMed: 11181812]

26. Rheault MN, Manivel JC, Levine SC, Sinaiko AR. Sarcoidosis presenting with hearing loss and granulomatous interstitial nephritis in an adolescent. Pediatr Nephrol. 2006; 21:1323-1326. [PubMed: 16810515]

*27. Pepple KL, Lam DL, Finn LS, Van Gelder R. Urinary $\beta 2$-microglobulin testing in pediatric uveitis: A case report of a 9-year-old boy with renal and ocular sarcoidosis. Case Rep Ophthalmol. 2015; 6:101-105. *Sarcoidosis masquerading as TINU. [PubMed: 25873895]

28. Iselin KC, Brandt S, Böni C. Bilateral uveitis and tubulointerstitial nephritis - an atypical case. Klin Monatsbl Augenheilkd. 2017; 234:575-576. [PubMed: 28264218]

29. Ikeda A, Nagai S, Kitaichi M, et al. Sarcoidosis with granulomatous interstitial nephritis: report of three cases. Intern Med. 2001; 40:241-245. [PubMed: 11310492]

30. Vargas F, Gedalia A, Craver RD, Matti Vehaskari V. Recurrence of granulomatous interstitial nephritis in transplanted kidney. Pediatr Transplant. 2010; 14:54-57.

**31. Joyce E, Glasner P, Ranganathan S, Swiatecka-Urban A. Tubulointerstitial nephritis: diagnosis, treatment, and monitoring. Pediatr Nephrol. 2017; 32:577-587. **Update on features, pathogenesis, and treatment of tubulointerstitial nephritis from nephrology perspective. [PubMed: 27155873]

32. Izzedine H. Tubulointerstitial nephritis and uveitis syndrome (TINU): a step forward to understanding an elusive oculorenal syndrome. Nephrol Dial Transplant. 2008; 23:1095-1097. [PubMed: 18272778]

33. Gafter U, Kalechman Y, Zevin D, et al. Tubulointerstitial nephritis and uveitis: association with suppressed cellular immunity. Nephrol Dial Transplant. 1993; 8:821-826. [PubMed: 8255514]

34. Yoshioka K, Takemura T, Kanasaki M, et al. Acute interstitial nephritis and uveitis syndrome: activated immune cell infiltration in the kidney. Pediatr Nephrol. 1991; 5:232-234. [PubMed: 2031842]

35. Abed L, Merouani A, Haddad E, et al. Presence of autoantibodies against tubular and uveal cells in a patient with tubulointerstitial nephritis and uveitis (TINU) syndrome. Nephrol Dial Transplant. 2008; 23:1452-1455. [PubMed: 18156461]

36. Tan Y, Yu F, Qu Z, et al. Modified C-reactive protein might be a target autoantigen of TINU syndrome. Clin J Am Soc Nephrol. 2011; 6:93-100. [PubMed: 20813859]

37. Li C, Su T, Chu R, et al. Tubulointerstitial nephritis with uveitis in Chinese adults. Clin J Am Soc Nephrol. 2014; 9:21-28. [PubMed: 24202135]

38. Mackensen F, David F, Schwenger V, et al. HLA-DRB $1 * 0102$ is associated with TINU syndrome and bilateral, sudden-onset anterior uveitis but not with interstitial nephritis alone. Br J Ophthalmol. 2011; 95:971-975. [PubMed: 21059595]

39. Peräsaari J, Saarela V, Nikkilä J, et al. HLA associations with tubulointerstitial nephritis with or without uveitis in Finnish pediatric population: a nation-wide study. Tissue Antigens. 2013; 81:435-441. [PubMed: 23594347]

40. Gorroño-Echebarría MB, Calvo-Arrabal MA, Albarrán F, Alvarez-Mon M. The tuberculointerstitial nephritis and uveitis (TINU) syndrome is associated with HLA-DR14 in Spanish patients. Br J Ophthalmol. 2001; 85:1010-1011. [PubMed: 11501522]

41. Gough SCL, Simmonds MJ. The HLA region and autoimmune disease: associations and mechanisms of action. Curr Genomics. 2007; 8:453-465. [PubMed: 19412418]

42. Cusick MF, Libbey JE, Fujinami RS. Molecular mimicry as a mechanism of autoimmune disease. Clin Rev Allergy Immunol. 2012; 42:102-111. [PubMed: 22095454]

43. Valencia X, Lipsky PE. CD4+CD25+FoxP3+ regulatory T cells in autoimmune diseases. Nat Clin Pract Rheumatol. 2007; 3:619-626. [PubMed: 17968332]

44. Yeh S, Li Z, Forooghian F, et al. CD4+Foxp3+ T-regulatory cells in noninfectious uveitis. Arch Ophthalmol. 2009; 127:407-413. [PubMed: 19365016]

45. Chen L, Yang P, Zhou H. Diminished frequency and function of CD4+CD25high regulatory T cells associated with active uveitis in Vogt-Koyanagi-Harada syndrome. Invest Ophthalmol Vis Sci. 2008; 49:3475-3482. [PubMed: 18421089] 
46. Ji S-R, Wu Y, Potempa LA, et al. Effect of modified C-reactive protein on complement activation: a possible complement regulatory role of modified or monomeric C-reactive protein in atherosclerotic lesions. Arterioscler Thromb Vasc Biol. 2006; 26:935-941. [PubMed: 16456095]

47. Sjöwall C, Bengtsson AA, Sturfelt G, Skogh T. Serum levels of autoantibodies against monomeric C-reactive protein are correlated with disease activity in systemic lupus erythematosus. Arthritis Res Ther. 2004; 6:87-94.

48. Lasave AF, You C, Ma L, et al. Long-term outcomes of rituximab therapy in patients with noninfectious posterior uveitis refractory to conventional immunosuppressive therapy. Retina. 2017 Feb 20. epub ahead of print.

49. Smith JR, Stempel AJ, Bharadwaj A, Appukuttan B. Involvement of B cells in non-infectious uveitis. Clin Transl Immunol. 2016; 5:e63.

50. Vohra S, Eddy A, Levin AV, et al. Tubulointerstitial nephritis and uveitis in children and adolescents. Four new cases and a review of the literature. Pediatr Nephrol. 1999; 13:426-432. [PubMed: 10412864]

51. Tanaka H, Suzuki K, Nakahata T, et al. Repeat renal biopsy in a girl with tubulointerstitial nephritis and uveitis syndrome. Pediatr Nephrol. 2001; 16:885-887. [PubMed: 11685594]

52. Yanagihara T, Kitamura H, Aki K, et al. Serial renal biopsies in three girls with tubulointerstitial nephritis and uveitis syndrome. Pediatr Nephrol. 2009; 24:1159-1164. [PubMed: 19277722]

53. Suzuki K, Tanaka H, Ito E, Waga S. Repeat renal biopsy in children with severe idiopathic tubulointerstitial nephritis. Pediatr Nephrol. 2004; 19:240-243. [PubMed: 14669098]

54. Murtaza A, Ali H, Nair B, Ahmed AK. Recurrence of tubulointerstitial nephritis without uveitis, in a patient with previous tubulointerstitial nephritis and uveitis syndrome after transplant. Exp Clin Transplant. 2016 Jun 29. epub ahead of print.

55. Jabs DA, Rosenbaum JT, Foster CS, et al. Guidelines for the use of immunosuppressive drugs in patients with ocular inflammatory disorders: recommendations of an expert panel. Am J Ophthalmol. 2000; 130:492-513. [PubMed: 11024423] 


\section{Key Points}

- Tubulointerstitial nephritis and uveitis (TINU) is a rare but important cause of uveitis in children and some adults.

- The "classic" TINU presentation is a bilateral simultaneous or sequential symptomatic non-granulomatous anterior uveitis in a pediatric patient.

- $\quad$ Recent data indicates a wider range of presentations can be manifestations of TINU, including intermediate and posterior disease, and presentation in adults.

- Diagnosis can be facilitated by targeted clinical testing, particularly elevated urinary beta-2 microglobulin. Diagnosis is supported by renal biopsy when indicated, and pathologic evaluation of the biopsy specimen can be particularly useful in ruling out important mimics.

- The prognosis for vision and renal function is generally good. Systemic steroid therapy results in prompt renal recovery in most cases and may provide the benefit of reduced uveitis recurrences. 
Table

Diagnostic criteria of tubulointerstitial nephritis and uveitis (adapted from Mandeville et al)[5].

Level of diagnostic certainty

• "Definite"

- Typical uveitis *

AND

- Interstitial nephritis on renal biopsy or by complete clinical criteria

- "Probable"

- Atypical uveitis and positive renal biopsy

OR

- Typical uveitis and incomplete interstitial nephritis clinical criteria

- "Possible"

- Atypical uveitis and incomplete interstitial nephritis clinical criteria

Interstitial nephritis by clinical criteria

- "Complete": all three criteria met

- "Incomplete": less than three criteria met
1. Abnormal renal function (elevated creatinine or decreased clearance)

2. Abnormal urinalysis (elevated $\beta 2$-microglobulin, low-grade proteinuria, pyuria, urinary eosinophils, hematuria, glycosuria, or white blood cell casts)

3. Systemic illness $\geq 2$ weeks (any combination of: signs and symptoms, such as fever, fatigue, weight loss; and laboratory abnormalities, such as anemia, abnormal liver function, eosinophilia, or elevated erythrocyte sedimentation rate)

Bilateral anterior uveitis with or without intermediate or posterior uveitis with an onset less than 2 months before or 12 months after the onset of interstitial nephritis. 